\title{
Convexification for data fitting
}

\section{James Ting-Ho Lo}

\section{Erratum to: J Glob Optim}

\section{DOI 10.1007/s10898-009-9417-z}

In the original publication under the section: 4 Convexity region, the Eqs. 6 and 7 reads as

$$
\begin{aligned}
& \sum_{k=1}^{K} \alpha_{k} B_{k}(w)=\mathcal{F}_{K}(w) \mathcal{B}_{K}(w) \mathcal{F}_{K}^{T}(w) \\
& \mathcal{F}_{K}(w):=\left[\begin{array}{lll}
F_{1}(w) & \cdots & F_{K}(w)
\end{array}\right] \\
& \mathcal{A}_{K}(w):=\operatorname{diag}\left[\alpha_{1}(w) Q \quad \cdots \quad \alpha_{K}(w) Q\right]
\end{aligned}
$$

and

$$
\begin{aligned}
& \sum_{k=1}^{K} \alpha_{k} A_{k}(w)=\mathcal{D}_{K}(w) \mathcal{A}_{K}(w) \mathcal{D}_{K}^{T}(w) \\
& \mathcal{D}_{K}(w):=\left[\begin{array}{lll}
D_{1}(w) & \cdots & D_{K}(w)
\end{array}\right] \\
& \mathcal{A}_{K}(w):=\operatorname{diag}\left[\alpha_{1}(w) \quad \cdots \quad \alpha_{K}(w)\right]
\end{aligned}
$$

are incorrect.

The correct version of Eqs. 6 and 7 are given below.

$$
\begin{aligned}
& \sum_{k=1}^{K} \alpha_{k}(w) B_{k}(w)=\mathcal{F}_{K}(w) \mathcal{B}_{K}(w) \mathcal{F}_{K}^{T}(w) \\
& \mathcal{F}_{K}(w):=\left[\begin{array}{lll}
F_{1}(w) & \cdots & F_{K}(w)
\end{array}\right] \\
& \mathcal{B}_{K}(w):=\operatorname{diag}\left[\alpha_{1}(w) Q \quad \cdots \quad \alpha_{K}(w) Q\right]
\end{aligned}
$$

The online version of the original article can be found under doi:10.1007/s10898-009-9417-z.

J. T.-H. Lo $(\bowtie)$

Department of Mathematics and Statistics, University of Maryland Baltimore County, Baltimore, MD 21250, USA

e-mail: jameslo@umbc.edu 
and

$$
\begin{aligned}
& \sum_{k=1}^{K} \alpha_{k}(w) A_{k}(w)=\mathcal{D}_{K}(w) \mathcal{A}_{K}(w) \mathcal{D}_{K}^{T}(w) \\
& \mathcal{D}_{K}(w):=\left[\begin{array}{lll}
D_{1}(w) & \cdots & D_{K}(w)
\end{array}\right] \\
& \mathcal{A}_{K}(w):=\operatorname{diag}\left[\alpha_{1}(w) \quad \cdots \quad \alpha_{K}(w)\right]
\end{aligned}
$$

